\title{
Le silence des anneaux : une tentative de reconstitution d'une chaîne opératoire de fabrication d'anneaux en os
}

The silence of the rings: an attempt to reconstructing a production process of bone rings

\section{Bénédicte Khan et Christophe Picod}

\section{(2) OpenEdition}

\section{Journals}

\section{Édition électronique}

URL : http://journals.openedition.org/artefact/1155

DOI : 10.4000/artefact. 1155

ISSN : 2606-9245

Éditeur :

Association Artefact. Techniques histoire et sciences humaines, Presses universitaires du Midi

\section{Édition imprimée}

Date de publication : 30 mai 2018

Pagination : 79-94

ISBN : 978-2-7535-7494-6

ISSN : 2273-0753

\section{Référence électronique}

Bénédicte Khan et Christophe Picod, «Le silence des anneaux : une tentative de reconstitution d'une chaîne opératoire de fabrication d'anneaux en os », Artefact [En ligne], 7 | 2017, mis en ligne le 29 janvier 2019, consulté le 06 mars 2020. URL : http://journals.openedition.org/artefact/1155; DOI : https://doi.org/10.4000/artefact.1155 


\section{Le silence des anneaux: une tentative de reconstitution d'une chaîne opératoire de fabrication d'anneaux en os}

Bénédicte KHAN*
Christophe PICOD

\section{Résumé}

$\mathrm{Au}$ Proche-Orient hellénistique et romain, des anneaux en os et en écaille de tortue sont produits à partir de plaquettes quadrangulaires. Cette production par plaquettes a soulevé des interrogations quant à sa réalisation. Cet article présente une reconstitution de sa chaîne opératoire de fabrication. Certaines séquences ont été déduites de l'étude des stigmates visibles sur les rejets artisanaux; d'autres découlent des connais-

*. Bénédicte Khan est doctorante à l'université Paris 1 Panthéon-Sorbonne sous la direction de Jacqueline Dentzer-Feydy et de Marianne Christensen. Dans le cadre de sa thèse, elle étudie l'artisanat des matières dures animales au Proche-Orient du III $^{\mathrm{e}}$ siècle av. J.-C. au vII ${ }^{\mathrm{e}}$ siècle apr. J.-C., principalement à partir d'une approche technologique. Elle a publié « L'artisanat des matières dures animales : nouvelles connaissances à partir des rebuts d'atelier », in Sarah FerJani, Amélie Le BiHAN, Marilyne Onfray et Caroline Trémeaud (éd.), Archéo.doct. 6, Matières premières et gestion des ressources. Actes de la $7^{e}$ Journée doctorale d'archéologie, Paris, 23 mai 2014, Paris, Publications de la Sorbonne, 2014, p. 143-161, en ligne : [http:/ / books.openedition.org/psorbonne/5099], mis en ligne le 14 décembre 2016. Contact : [benedicte. khan@gmail.com].

**. Christophe Picod est issu d'une famille de tourneurs sur bois du Jura depuis plusieurs générations. Tourneur amateur, il publie ses recherches à la fois historiques et techniques sur le tournage et le métier de tourneur depuis 1990. Il travaille en collaboration avec des archéologues avec lesquels il monte des expérimentations sur les manières de faire antiques et médiévales sur os et bois de cervidé. Il a publié, avec Michel Feugère, " Reconstitution de dés en matière dure animale, antiques et médiévaux », Instrumentum, 39, 2014, p. 37-41; avec Isabelle Rodet-BELARBi et Madeleine CHÂTELET, « La fabrication des peignes en bois de cerf et en os de l'Antiquité tardive et du haut Moyen Âge : étude tracéologique et expérimentation sur les peignes d'Obernai et de Marlenheim (Bas-Rhin) », Instrumentum, 44, 2016, p. 36-45. Contact : [cpicod@orange.fr].

Les auteurs souhaitent remercier Hervé Monchot pour son aide lors de l'identification des restes fauniques liés au travail de l'os dans le matériel du Qasr al-Bint. De même, leurs profonds remerciements vont à François Renel, Christian Augé (†) et Laurent Tholbecq, pour leur avoir offert d'étudier le matériel du Qasr al-Bint, à l'origine de cette démarche expérimentale, ainsi qu'à Annegret Schneider et Jacqueline Studer pour avoir si gentiment partagé leurs données sur le matériel d'ez-Zantur. Enfin, ils souhaitent remercier Steven Sidebotham et Iwona Zych d'avoir accepté qu'ils étudient, en partenariat avec Mariusz Gwiazda, le matériel en écaille de tortue de Béréniké. 
sances sur l'outillage aux époques étudiées et de la mise en pratique de techniques lors de l'expérimentation.

Mots-clés : anneau, artisanat, expérimentation, Proche-Orient, technologie.

\section{Abstract. The silence of the rings : an attempt to recons- tructing a production process of bone rings}

Bone and turtleshell rings made from quadrangular plaques were unearthed on different near-eastern sites from the Hellenistic and Roman times. This chaine opératoire raised several questions, especially on how the production was carried out. This article presents a replication of a ring production process. The study of the working refuse allowed the deduction of some of the stages of work. Informations on the toolkit available to the Ptolemaic and Roman craftsmen also helped direct the experimental tries.

Key-words : craftsmanship, experimentation, Near East, ring, technology.

De la fin du $\mathrm{III}^{\mathrm{e}}$ siècle av. J.-C. au $\mathrm{V}^{\mathrm{e}}$ siècle apr. J.-C. au moins, de Béréniké au sud de l'Égypte à Césarée-Maritime en Israël et jusqu'à Carthage en Tunisie, les artisans produisirent des anneaux en os et, dans une moindre mesure, en écaille de tortue et en corne ${ }^{1}$. Ces anneaux n'ont pour l'instant été retrouvés qu'en contextes artisanaux, ateliers ou zones de rejets secondaires. Aussi, leur fonction reste indéterminée. On a supposé qu'il s'agissait d'éléments de parure, des perles, des bagues ou des anneaux d'attache pour les vêtements ${ }^{2}$, d'éléments utilitaires, comme des œillets ou des éléments de fermeture pour sacs ou bourses $^{3}$, ou encore d'objets en lien avec le travail du textile, pour le filage de la laine ${ }^{4}$.

Les rebuts d'ateliers ont permis d'identifier au moins deux chaînes opératoires de fabrication différentes : l'une exploitant la forme naturellement tubulaire de la diaphyse d'un os long et déjà amplement étudiée ${ }^{5}$, la seconde à partir de plaquettes quadrangulaires en os ou en écaille de tortue préalablement extraites $^{6}$. Cette dernière, qui fait l'objet de cet article, a été relevée sur les sites du Qasr al-Bint à Pétra et à Béréniké, où nous avons conduit une analyse technologique.

Le matériel du Qasr al-Bint est composé de quarante-huit objets en os associés de manière certaine à une production d'anneaux sur plaquettes, datés de la fin du Ive-début du v siècle apr. J.-C. À Béréniké, les soixantedeux rejets sont en écaille de tortue et datent des périodes ptolémaïque (fin $\mathrm{IV}^{\mathrm{e}}$ siècle-30 av. J.-C.) et romaine tardive (IV ${ }^{\mathrm{e}}-\mathrm{VI}^{\mathrm{e}}$ siècle apr. J.-C.).

Toutes les étapes de production ne sont pas représentées dans le matériel 
archéologique, parce qu'elles n'ont pas marqué la matière ou parce qu'elles ont été effacées par les étapes suivantes. Les sources textuelles ne semblant pas pouvoir pallier ce manque ${ }^{7}$, nous avons tenté une approche expérimentale. Le protocole de cette expérimentation a été élaboré à partir des stigmates identifiés sur les objets semi-finis et sur les rebuts de production, ainsi que sur le panel d'outils connus aux périodes concernées, pour approcher au plus près les manières de faire des époques hellénistique et romaine tardive.

\section{Les traces d'outils, révélatrices des techniques employées}

L'étude a été effectuée en suivant une approche instaurée par des chercheurs en Préhistoire. Elle s'attache à l'identification des techniques et des procédés techniques mis en place à partir des traces d'outils ${ }^{8}$. L'agencement de ces marques techniques permet de reconstituer la chronologie des différentes étapes formant la chaîne opératoire de production. Fondée sur une lecture des stigmates et donc du geste, cette méthodologie peut s'appliquer à des objets de toutes périodes : l'arrivée du métal n'a pas forcément changé les façons de faire, souvent dictées par la matière première et sa structure. $D^{\prime}$ une manière générale, l'utilisation d'outils en métal dans le milieu artisanal a surtout permis d'augmenter la vitesse et la précision de l'exécution'. L'outillage a peu évolué depuis, si ce n'est du fait des avancées dans le travail du métal en lui-même ${ }^{10}$ et de la motorisation.

Il nous paraît important de préciser qu'une technique, entendue ici comme " action élémentaire sur la matière ${ }^{11}$ ", n'est pas rattachée à un outil spécifique. Le lexique technique moderne, régulièrement employé par les chercheurs spécialistes des époques antique et plus tardives, lie implicitement les deux. Pourtant, plusieurs outils peuvent, en étant utilisés de la même façon, avoir laissé des traces similaires ${ }^{12}$, d'où le choix d'employer une terminologie développée par les préhistoriens, considérée comme plus neutre. Il est évident que des micro-différences peuvent apparaître de par l'utilisation d'outils différents; une étude expérimentale approfondie reste à mener pour les déterminer. 


\section{L'origine des matières premières employées}

Les anneaux ont été fabriqués en os et en écaille de tortue. L'os provient de grands mammifères herbivores domestiqués. À Pétra, les artisans ont exploité les os longs matures ou immatures, métapodes surtout, mais également tibias de camélidés (Camelus cf. dromedarius), et peut-être aussi de bovinés (Bos taurus ${ }^{13}$ ). Bien que les conditions de conservation ne permettent pas toujours d'affirmer avec certitude de quelles espèces provenaient les écailles de tortue travaillées sur le site de Béréniké, il semble que la tortue imbriquée (Eretmochelys imbri- cata) et peut-être aussi la tortue verte (Chelonia mydas) ont été les principales sources de matières premières pour la fabrication d'anneaux ${ }^{14}$.

La corne enfin semble avoir été employée à Béréniké ${ }^{15}$. La présence de chevilles osseuses de bovins et d'ovinscaprins dans le matériel du Qasr al-Bint peut aussi suggérer une production d'anneaux sur corne; cependant, aucun élément en corne n'ayant été conservé à Pétra, cette hypothèse ne peut être vérifiée.

\section{Les rejets de fabrication : description technique}

Les rebuts de la production d'anneaux sur plaquette en os (Fig. 1) sont des fragments de diaphyse, des plaquettes, perforées ou non, des bords de plaquettes après extraction d'un élément circulaire, et des pièces de contour circulaire perforées en leur centre, liées au découpage de la partie interne de l'anneau. Quelques anneaux incomplets font également partie du matériel. À l'exception des fragments de diaphyse pour des raisons évidentes, les rejets de fabrication d'anneaux en écaille de tortue sont similaires.

\section{Extrémités de plaque (Fig. 1.a)}

Deux éléments de diaphyse de section rectangulaire en leur extrémité distale portent des traces de travail (Fig. 1.a). L'extrémité proximale est souvent brute ou faiblement modifiée. Parfois, sur leur face inférieure, subsiste un reliquat de tissu spongieux, indiquant une pièce prélevée près des épiphyses. Deux types de stigmates sont observables : des plages de stries provenant d'un raclage dans l'axe longitudinal de l'os, et un pan de sillon perpendiculaire à cet axe, lié au sciage qui a segmenté l'os. Les stries sont interrompues par le sillon : le raclage est antérieur au sciage. Il y a donc préparation de la pièce avant sectionnement.

\section{Plaquettes (Fig. 1.b et c)}

Au nombre de treize au Qasr al-Bint, elles sont de contour et de section rectangulaires (Fig. 1.b). Elles ont une épaisseur moyenne de 5,5 à $6 \mathrm{~mm}$, mais qui peut varier entre 4 et $9 \mathrm{~mm}$, et une largeur entre 26 et $31,5 \mathrm{~mm}$. Les trois pièces entières ont une longueur com- 


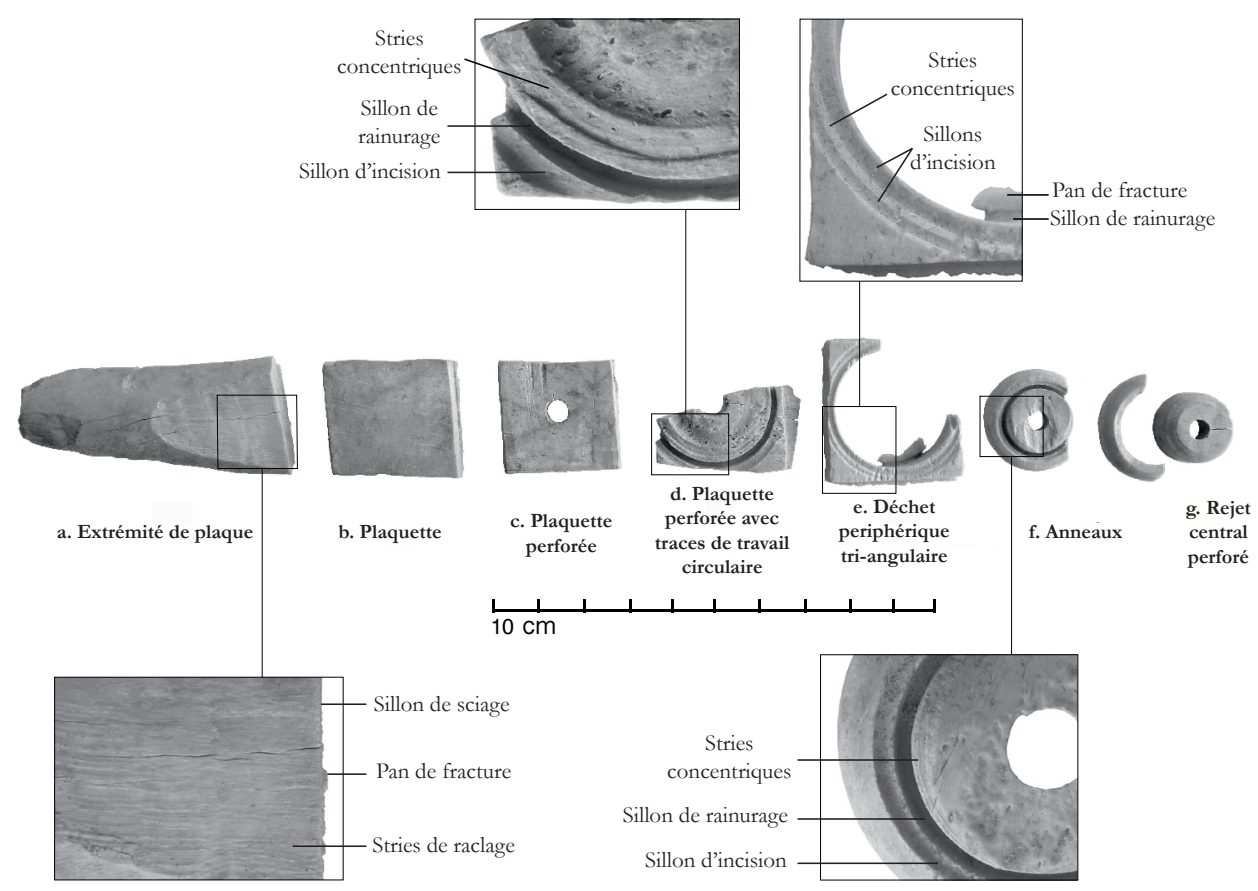

Fig. 1. - Rebuts osseux liés à la production d'anneaux sur plaquettes, organisés selon les différentes étapes de la chaîne opératoire de fabrication auxquelles ils sont rattachés. La figure 1.b peut ne pas être un déchet, mais un support qui n'a pas été utilisé au moment de son abandon pour diverses raisons inconnues. À l'inverse, la présence de sillons de sciage sur le bord supérieur gauche de la figure 1.c indique clairement qu'il s'agit d'un déchet. Ces sillons auraient été difficiles à supprimer sans réduire

l'épaisseur et/ou la largeur de l'anneau, le fragilisant d'autant. Clichés et DAO de l'auteur.

prise entre 28,5 et $32 \mathrm{~mm}$. Une seule plaquette en écaille de tortue a été retrouvée à Béréniké. De forme trapézoïdale et perforée en son centre, elle mesure $27 \mathrm{~mm}$ de long, $24 \mathrm{~mm}$ de large et 1,2 à $3 \mathrm{~mm}$ d'épaisseur.

Les plaquettes portent des plages de stries couvrant les deux faces, ainsi que les bords. Ces plages sont organisées de façon irrégulière en suivant le sens des fibres de l'os, attestant d'un raclage. Les extrémités des plaquettes, transversales par rapport à l'axe des fibres osseuses, présentent des pans de sillon résultant d'un sciage. Souvent une partie du fond de sillon est conservée. Elle peut être située soit sur la lèvre supérieure ou inférieure, signalant un sciage unifacial, soit vers le milieu, où deux pans de sillon opposés se rejoignent pour former une arête, indiquant un sciage bifacial. Un pan de fracture en languettes témoigne d'un procédé de détachement par flexion.

Six plaquettes du Qasr al-Bint portent approximativement en leur centre une perforation parfaitement cylindrique dont le diamètre mesure entre 3,9 et $4 \mathrm{~mm}$ (Fig. 1.c). À Béréniké, la perforation de la plaquette mentionnée plus haut est plus petite : elle fait $3,5 \mathrm{~mm}$ de diamètre. La perforation a été réalisée par un travail unifacial d'après le pan de sillon et les languettes d'arrachement. 
Plaquettes perforées avec traces de travail circulaires (Fig. 1.d)

Sur la face supérieure de deux plaquettes perforées (une sur chaque site), des plages de stries circulaires concentriques, peu profondes, très fines et parallèles entre elles, sont présentes sur le pourtour d'au moins un sillon circulaire vertical, plus profond (Fig. 1.d). Ces plages, que l'on retrouve aussi sur certains déchets périphériques angulaires (Fig. 1.e), ont été identifiées comme étant les stigmates d'une incision, ou plutôt d'un rainurage superficiel. Elles indiquent une préparation, un positionnement de l'outil, comme une sorte d'amorce avant de pénétrer plus profondément dans la matière. Les sillons profonds constituent les bords externe et interne du futur anneau sur la plaquette. Lorsque le fond est conservé, il présente un profil en $\mathrm{U}$, aux pans parallèles. La largeur moyenne de ces sillons est de $1 \mathrm{~mm}$. Des stries fines, longues et parallèles entre elles sont visibles sur les pans, permettant d'identifier un rainurage unifacial. On note des pans de fracture en languettes sur le bord du fond de sillon, indiquant un détachement de la pièce circulaire centrale par flexion, probablement dû à la pression exercée par l'outil.

\section{Déchets périphériques angulaires (Fig. 1.e)}

Il s'agit des parties angulaires de la plaquette après extraction de l'anneau (Fig. 1.e). Les pièces ont un pan de délinéation concave produit par un rainurage unifacial circulaire, et des pans droits, qui sont les bords externes de la plaquette, produits soit par sciage soit par raclage. Les faces supérieure et inférieure présentent également des traces de raclage. Les déchets périphériques angulaires peuvent être simples, c' est-àdire que le rejet comptabilise un angle de la plaquette originelle, ou bi-angulaires, quand deux angles sont encore associés; ils peuvent également être tri-angulaires, quand une pièce possède encore trois angles complets. On comptabilise seize déchets simples, trois biangulaires et un tri-angulaire au Qasr al-Bint. À Béréniké, on compte vingtcinq déchets simples, sept bi-angulaires et un tri-angulaire. Aucun rebut lié à la fabrication d'anneau par plaquette ne présente quatre angles après extraction de l'anneau. Cela peut s'expliquer par le fait que les artisans ont cherché à obtenir le plus grand anneau possible entièrement contenu dans la plaquette. Ils ont donc pu tracer le cercle externe si près $\mathrm{du}$ bord que, lorsque la partie externe de l'anneau a été mise en place, le bord de la plaquette a été si affiné qu'il s'est désolidarisé. De plus, il est possible que la perforation ne soit pas parfaitement centrale et que la plaquette ne soit pas parfaitement carrée. Cela a pu entraîner un léger décalage, avec un bord légèrement plus court que l'autre par rapport à la perforation, et avoir pour effet de devoir travailler très près du bord plus court.

\section{Anneaux (Fig. 1.f)}

$\mathrm{Au}$ Qasr al-Bint, quatre anneaux incomplets de deux différents diamètres ont été retrouvés (Fig. 1.f). Les trois plus grands font environ $22 \mathrm{~mm}$ de diamètre 
externe et $17 \mathrm{~mm}$ de diamètre interne. Le plus petit fait $16 \mathrm{~mm}$ de diamètre extérieur et $14 \mathrm{~mm}$ de diamètre intérieur. À Béréniké, trois anneaux conservés font entre 18 et $17 \mathrm{~mm}$ de diamètre externe et entre 14 et $13 \mathrm{~mm}$ de diamètre interne. Deux anneaux font entre 15 et $14 \mathrm{~mm}$ de diamètre extérieur et $11 \mathrm{~mm}$ de diamètre intérieur. Trois anneaux de diamètre beaucoup plus large, pouvant atteindre $60 \mathrm{~mm}$ de diamètre externe et $50 \mathrm{~mm}$ de diamètre interne, ont également été mis au jour.

Les anneaux conservés, qui ont sûrement cassé pendant leur extraction ou pendant les étapes de finition, présentent une section plano-convexe et le pourtour extérieur est poli; la face interne porte les traces du rainurage.

\section{Rejets centraux perforés (Fig. 1.g)}

Ces pièces sont des éléments circulaires perforés dont l'orifice central cylindrique est de même dimension que celui des plaquettes (Fig. 1.g et c). $\mathrm{Au}$ Qasr al-Bint, cinq rejets centraux mesurent entre 13 et $15,5 \mathrm{~mm}$ de diamètre, deux entre 9 et $11 \mathrm{~mm}$ de diamètre. Elles ont toutes une épaisseur normalisée située entre 4 et $5 \mathrm{~mm}$, ce qui est légèrement inférieur aux dimensions des plaquettes (5,5 à $6 \mathrm{~mm}$ en moyenne). Cette différence peut se justifier par un travail de préparation autour de la perforation avant creusement des sillons et/ou du fait de la friction de l'outil qui a pu, lors du rainurage, accrocher la surface et enlever de la matière de façon fortuite. À Béréniké, les vingt-deux pièces ont un diamètre compris entre $9,4 \mathrm{~mm}$ et $15 \mathrm{~mm}$. Une seule pièce atteint $19 \mathrm{~mm}$ de diamètre. Elles font entre 1 et $4,4 \mathrm{~mm}$ d'épaisseur; mais beaucoup ne sont pas entières dans leur épaisseur, ce qui explique un tel écart entre les pièces.

Le bord extérieur des rejets centraux est un pan de sillon. Tout ou partie du fond de sillon, qui est en $U$, peut être conservé sur le bord inférieur de la pièce, et porter des stigmates de détachement par flexion. Parfois, un deuxième fond de sillon est visible sur le bord externe, séparé du pan et du fond de sillon par un pan de fracture, produit au moment du détachement de l'anneau (Fig. 2.a).

Quatre incisions verticales formant les angles d'un carré sont visibles de façon plus ou moins prononcée sur les lèvres des perforations centrales. Elles se superposent aux stries à l'intérieur des perforations, indiquant qu'elles ont été réalisées postérieurement (Fig. 2.b). Parce qu'elles portent des traces d'usure et figurent également sur quelques plaques perforées présentant des amorces de travail circulaire, ces inci-
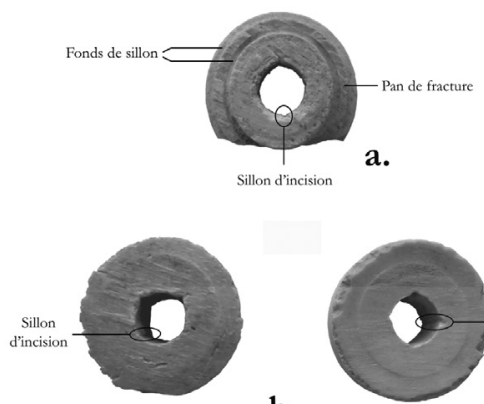

b.

Fig. 2. - Déchets centraux présentant deux fonds de sillons circulaires sur le pourtour (a.), ainsi que des incisions verticales dans la perforation (a. et b.). Clichés et DAO de l'auteur. 
sions amènent à réfléchir sur l'existence d'un élément quadrangulaire inséré dans la perforation. Cet élément ser- virait au maintien de la pièce en place lorsqu'elle est travaillée par rainurage.

\section{Reconstitution de la chaîne opératoire}

L'agencement des stigmates sur les pièces, ainsi que les liens évidents existant entre les différentes catégories d'objets que nous venons de présenter, nous ont permis de proposer une hypothèse de reconstitution de chaîne opératoire (Fig. 3). Il semble que l'artisan ait travaillé l'os comme suit :

1. Suppression par sciage des épiphyses des os longs pour récupérer la diaphyse.

2. Débitage longitudinal de la diaphyse afin $\mathrm{d}^{\prime}$ obtenir des plaques
3. Façonnage de la diaphyse par raclage, dans le but d'obtenir des surfaces planes et régularisées, lui conférant une section approximativement rectangulaire.

4. Subdivision de la grande plaque en plusieurs plaquettes quadrangulaires de dimensions similaires par sciage transversal. Ne sont segmentées que les portions présentant les conditions requises pour leur utilisation en tant que support à anneau : épaisseur suffisante, tissu spongieux limité ou inexistant, etc.

5. Perforation cylindrique des plaquettes à peu près en leur centre. rement identifiée sur le matériel archéologique).
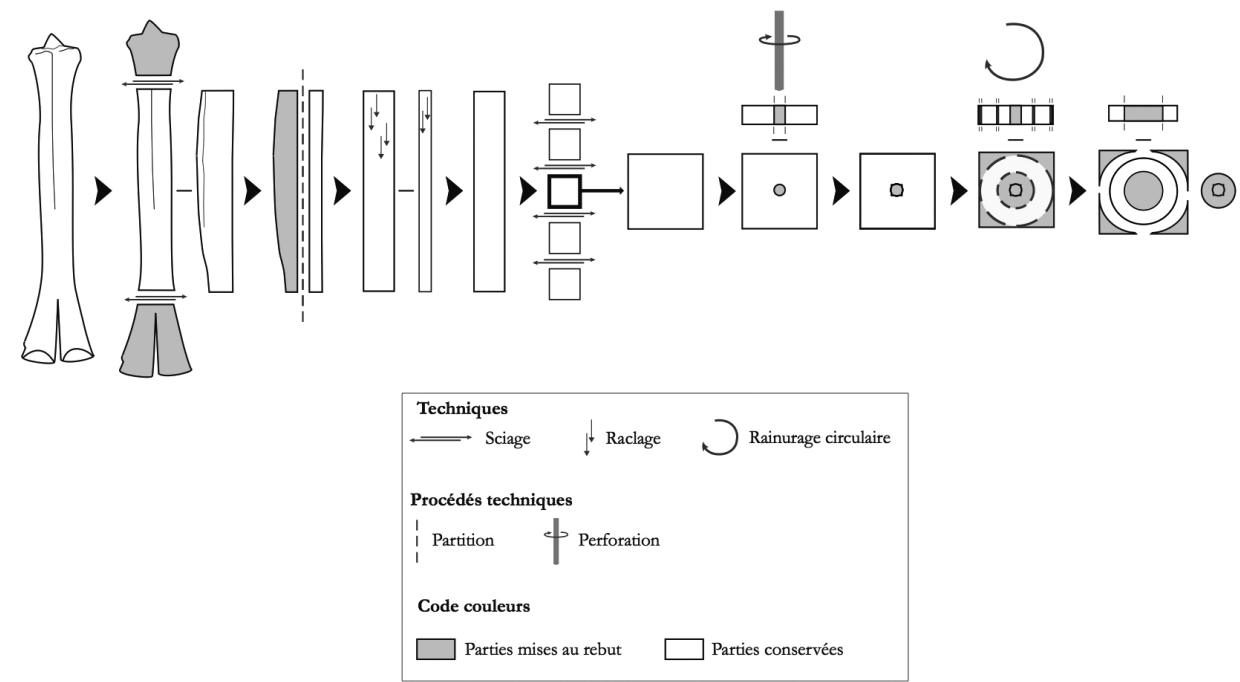

Fig. 3. - Hypothèse de reconstruction de la chaîne opératoire de production d'anneaux sur plaquettes à partir d'un métapode de dromadaire. DAO de l'auteur. 
6. Aménagement des incisions à l'intérieur de la perforation centrale.

7. Découpage de l'anneau dans la plaquette. La plaquette est rainurée unifacialement de manière circulaire, d'abord de manière superficielle pour vérifier que l'anneau est entièrement compris dans la plaquette, puis, une fois le diamètre calé, en profondeur, creusant un sillon en $U$, jusqu'au détachement du pourtour de l'anneau (déchet périphérique) et du rejet central perforé.

On ne sait pas précisément quand le sillon permettant le dégagement de la partie interne de l'anneau est établi. Une des plaquettes conservées semble indiquer une mise en place dans le même temps que le sillon externe. Néanmoins, la présence du poli sur la partie extérieure d'un anneau encore rattaché au rejet central perforé - où le sillon interne est déjà bien profond - pose la question d'un détachement préalable de la partie externe avant la partie interne (Fig. 1.f).
Pour l'écaille de tortue, le schéma opératoire suit le même principe. Cependant, les étapes de préparation de la plaquette sont moins bien connues. Il semble qu'après une étape de raclage de la face supérieure, les plaquettes ont été débitées sur au moins trois côtés par sciage (Fig. IX, cahier couleur).

Les objets mis au jour dans les zones de rejet permettent de reconnaître différentes étapes de la chaîne opératoire de production d'anneaux à partir de plaquettes en matières dures d'origine animale. Toutefois, leur mise en place reste hypothétique. C'est pourquoi il a été jugé nécessaire de reconstituer la chaîne opératoire de manière expérimentale. Pour ce faire, nous avons collaboré avec un tourneur expérimental, Christophe Picod. Plusieurs éléments furent discutés. Avant toute chose, il fallait déterminer quels étaient les outils utilisés aux époques concernées concordant avec les informations obtenues par la lecture technique des rebuts de production.

\section{L'outillage à disposition : rapide bilan des sources}

La quasi-absence d'outils liés de manière formelle ${ }^{16}$ au travail des matières animales nous a amené à nous tourner vers le travail du bois, où l'équipement identifié est plus conséquent. Les matières dures d'origine animale et le bois, surtout le bois dur, semblent avoir été façonnés de la même manière ${ }^{17}$ avec les mêmes outils ${ }^{18}$. Il est tout à fait envisageable que ces matériaux aient été travaillés dans les mêmes ateliers, ou du moins dans les mêmes zones artisanales, du fait de leur complémentarité : charnières en os et pièces de placage pour le mobilier ${ }^{19}$, etc.

Pour les étapes de préparation et de mise en forme du support, l'outillage est bien connu : scies, planes à simple et double poignée, herminettes, ciseaux, gouges, limes, écouanes ont été retrouvés sur divers sites hellénistiques et romains. Ils sont également présents dans les sources textuelles, épigraphiques et iconographiques ${ }^{20}$. 
L'existence de la tarière et du foret, utilisés pour les perforations, est établie à l'époque romaine ${ }^{21}$. Bien que le trépan à archet ne soit pas cité en tant que tel dans les textes, il est représenté à Pompéi sur une peinture murale datant de 65 apr. J.-C ${ }^{22}$. Les mèches à pointes multiples sont déjà employées à ces époques, mises en mouvement à l'aide $\mathrm{d}^{\prime}$ un $\operatorname{archet}^{23}$. Pline $\mathrm{l}^{\prime}$ Ancien notamment cite le tour en bois ${ }^{24}$. Cet outil, connu dès le ve siècle av. J.-C. dans les textes, mais non décrit, n'a jamais été retrouvé en fouilles et est rarement figuré; on peut citer la représentation d'un tour actionné par un archet sur un sarcophage romain incomplet retrouvé en Grèce ${ }^{25}$. La scie cylindrique type cloche existait également dans l'Antiquité, comme l'attestent les exemplaires retrouvés à Bliesbruck du ${ }^{\text {er }}$ siècle apr. J.-C. ${ }^{26}$, et à Bingen de la fin du $\mathrm{I}^{\mathrm{er}}$-première moitié du $\mathrm{II}^{\mathrm{e}}$ siècle apr. J.-C. ${ }^{27}$.

\section{L'outillage expérimental, les raisons d'un choix}

La finesse des sillons observés sur les plaquettes rectangulaires ayant un sillon circulaire (1 $\mathrm{mm}$ de large), leur rectitude et le parallélisme de leurs pans laire pénétrant verticalement la matière comme la scie cylindrique type cloche. Cependant, la présence des plages de stries concentriques autour des sillons (Fig. 1.d et e) indique plutôt l'emploi d'un outil au diamètre non calibré. De plus, avec un outil calibré, la perforation centrale serait conique et non traversante $^{28}$. De fait, l'utilisation d'une scie cylindrique type cloche, dont le diamètre est fixe, ne peut être envisagée.

Si l'on emploie une mèche à pointes multiples où la pointe centrale est plus longue, la perforation centrale est conique et ne présente pas les incisions verticales relevées sur le matériel archéologique étudié29. Et, de même que pour la scie cylindrique type cloche, une mèche à pointes multiples ne peut créer que des diamètres fixes. Si un tel outil avait été utilisé, on ne trouverait pas non plus de stries concentriques autour des sillons; les stries obtenues par le calage de la mèche sur la plaquette se superposeraient, car alors elles seraient de même diamètre. Cette hypothèse a donc également été écartée.

Nous sommes arrivés à la conclusion qu'une lame fine biseauté ${ }^{30}$ devait avoir été utilisée pour creuser un sillon et extraire l'anneau de la plaquette. Pour obtenir un produit semi-fini circulaire, il faut que l'outil, ou la plaquette, soit mis en mouvement circulairement. De fait, le postulat d'un travail au tour s'est imposé. Les exemples ethnologiques connus montraient que le support était mis en rotation et que l'outil était tenu sur une barre parallèle à l'axe longitudinal du tour installée sur l'un de ses côtés ${ }^{31}$. C'est pourquoi nous nous sommes arrêtés sur l'idée d'une giration de la plaquette.

Le tour que l'on suppose avoir été utilisé est un tour à pointes, possédant deux poupées dont l'une est mobile. Chaque poupée présente une tige métallique 
appointée (la pointe) entre lesquelles vient s'installer l'élément à façonner ${ }^{32}$. Le rainurage, technique par définition unidirectionnelle ${ }^{33}$, indique que la plaquette n'est travaillée que dans un sens, lorsque qu'elle tourne vers l'artisan ${ }^{34}$. Par conséquent, il a été admis que le tour utilisé était à rotation alternative et fonctionnant à l'aide d'un archet, qui ne permet de façonner la matière que dans un sens ${ }^{35}$.

Les incisions verticales des perforations centrales nous ont mené à imaginer la plaquette maintenue sur un mandrin à l'aide d'une cheville de section carrée. L'utilisation du mandrin permet de proposer une surface large contre laquelle la plaquette peut être maintenue. Cela limite les risques d'un décrochage lors de la mise en mouvement et permet de mieux répartir la pression subie par la plaquette pendant le travail. C'est autour du mandrin que la corde de l'archet va s'enrouler et lancer la rotation.

\section{Déroulement de l'expérimentation}

Il a été décidé de se focaliser sur les étapes d'extraction de l'anneau. L'expérimentation fut menée à partir de plaquettes perforées en os déjà préparées de manière moderne, à la scie et au foret. Ces plaquettes ont été obtenues à partir de plaques d'os long de bœufs, radius ou tibia, provenant de restes de boucherie, dont les épiphyses avaient été préalablement éliminées. Les os avaient été bouillis dans un mélange d'eau et de soude, avant d'être mis à tremper dans un bain de trichloréthylène pour débarrasser la matière première de toute graisse ${ }^{36}$. Il est peu aisé de forcer l'insertion d'un élément dans l'os sans risquer de le briser; de fait, les quatre incisions verticales ont été aménagées dans la perforation à l'aide d'une lime. Un tour à pointes reconstitué (Fig. 4.a) a été utilisé pour les étapes de tournage ${ }^{37}$. Une gouge et un bédane ont été employés pour araser la surface de la plaquette avant le rainurage et faciliter celui-ci. Cette étape s'avère nécessaire à l'expérimentation : bien que le raclage

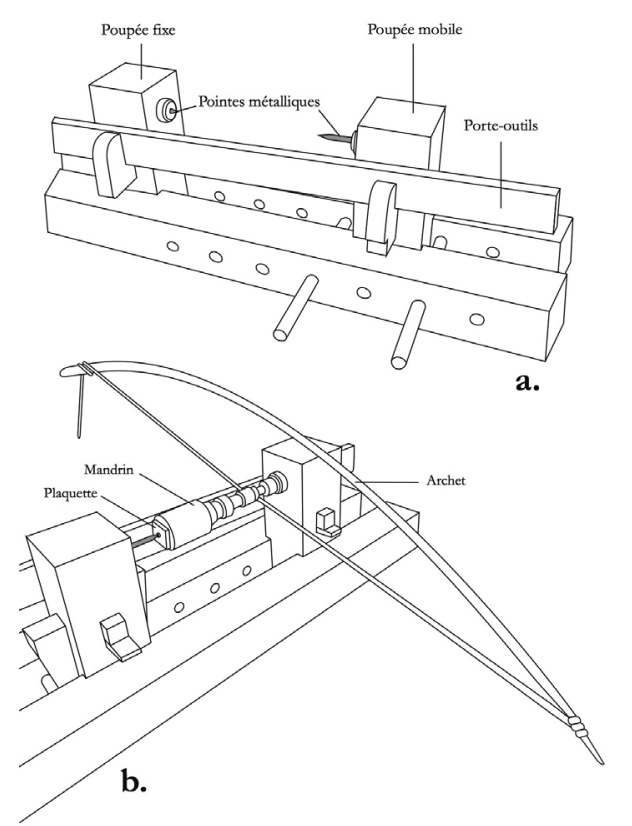

Fig. 4. - Représentation du tour expérimental de Christophe Picod avec une vue face à l'artisan (a.), et une vue arrière par rapport à l'artisan, une fois le mandrin installé sur le tour (b.). DAO de l'auteur. 
préliminaire ait permis d'aplanir la face supérieure de la plaquette, celle-ci est loin d'être parfaitement régularisée, ce qui peut poser problème lors du rainurage. La partie active de l'outil servant à rainurer a été préparée dans un coupoir

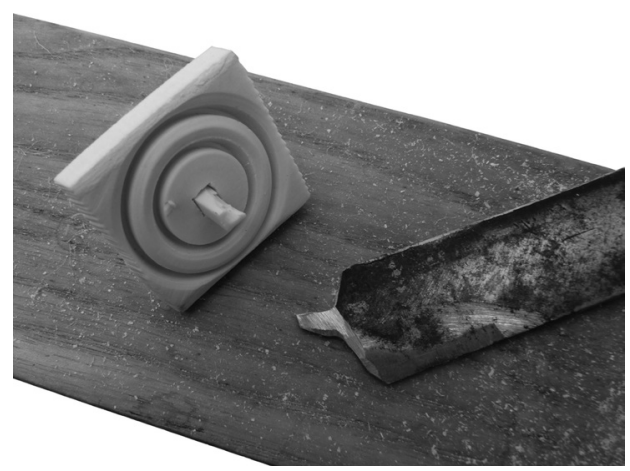

Fig. 5. - Extraction de l'anneau en cours d'expérimentation : plaquette en os après que la cheville en os a cassé; à droite, l'outil préparé sur coupoir. Cliché de l'auteur.

à lame très fine, sous forme de crochet (Fig. 5).

La plaquette en os est d'abord laissée vingt-quatre heures à tremper dans de l'eau afin de faciliter la pénétration de l'outil dans la matière. Elle est ensuite installée sur le mandrin dont le centre est creusé de façon quadrangulaire pour accueillir la cheville. Celle-ci doit être suffisamment longue pour traverser entièrement la plaquette et s'enfoncer dans le mandrin et être assez solide pour résister à la pression du tournage sans casser. La plaquette est calée à l'aide de la cheville, en hêtre lors du premier essai, et de trois petites pointes métalliques installées sur le mandrin, servant de butées $^{38}$. Elles limitent les oscillations de la plaquette et évitent qu'elle ne tourne sur elle-même lorsqu'elle est mise en mouvement. Le mandrin est positionné entre les deux pointes du tour (Fig. 4.b). La pointe de la poupée mobile est appuyée contre la cheville; le centre de la plaquette se situe ainsi dans l'axe longitudinal formé par les pointes. La poupée mobile est alors bloquée à l'aide d'un coin, afin que l'ensemble puisse résister à la pression du tournage sans que le mandrin se décale.

Le tour est mis en mouvement. La gouge est posée sur une barre parallèle à l'axe longitudinal du tour et des pointes, placée contre les poupées. Cette barre en bois, appelée porte-outil, offre un appui sur lequel l'outil peut être maintenu lors du travail, et permet d'éviter le décalage du sillon. Une fois la surface plane, le coupoir vient rainurer la matière de manière superficielle jusqu'à atteindre le diamètre permettant à la partie externe de l'anneau d'être entièrement comprise dans la plaquette. Dans les deux cas, l'outil ne s'approche de la plaquette que lorsque celle-ci tourne vers l'artisan. Lorsque le bon diamètre est trouvé, le coupoir s'enfonce dans la matière, aménageant un sillon de délinéation circulaire. Quand le sillon externe est bien marqué, le sillon délimitant la partie interne de l'anneau est mis en place. Si les deux sillons ne sont pas travaillés dans le même temps, les angles de la plaquette se détachent une fois que le sillon externe a traversé la plaquette. L'ébauche n'est alors plus tenue que par la cheville centrale, les pointes métalliques n'étant plus là pour aider à stabiliser la pièce. Cela entraîne une plus grande pression sur la cheville qui présente un plus grand risque de casser.

Rapidement l'outil utilisé patine, la lame vibre; il devient très difficile de 
creuser plus en avant la matière. Il faut forcer sur le coupoir et élargir le sillon afin de tenter d'aller plus profondément. La cheville en hêtre casse vite, du fait de la pression exercée par la pointe de la poupée mobile. L'ensemble se disloque. Un second essai est réalisé à l'aide d'une cheville en os. Pour éviter que le coupoir ne patine à nouveau, la partie active est retaillée et des affûtages réguliers opérés. Malgré cela, à partir d'une certaine profondeur, le crochet se coince dans le sillon. Il faut forcer à nouveau, mais le mandrin se décentre et la cheville en os se brise (Fig. 5).

Après réflexion, l'idée d'une partie active en forme de crochet est abandonnée. L'outil est façonné dans une lime, plus épaisse que le coupoir. L'épaisseur permet, en plus de limiter les vibrations causées par une trop grande finesse de la lame, d'instaurer une dépouille et un profil légèrement courbé pour éviter les broutages, tout en gardant une partie active fine. Avec la mise en place de la dépouille, l'arrière de la partie active est plus fine que l'avant, ce qui limite les risques que l'outil se coince dans le sillon. Une cheville en bronze est employée pour le troisième essai. Ce dernier s'avère plus concluant que les deux premiers. Toutefois, en dépit d'une plus grande stabilité apportée par la cheville en bronze, il devient particulièrement difficile à partir d'une certaine profondeur de creuser plus avant. Il faut élargir le sillon qui perd son profil en U. Alors il est possible de traverser presque entièrement l'épaisseur de la plaquette. Celle-ci est détachée du tour et l'anneau est extrait manuellement à l'aide d'un marteau et d'un tournevis utilisé comme coin, laissant sur le bord externe de l'anneau des languettes de détachement. Un dernier essai est réalisé sur une plaquette en corne. Il s'agit de se rendre compte des différences entre le travail de l'os et celui de la kératine dont sont constituées la corne et l'écaille de tortue ${ }^{39}$. La kératine est plus tendre : l'outil pénètre plus aisément et plus en profondeur que dans l'os. Toutefois, il faut également vers la fin du rainurage élargir celui-ci pour traverser les derniers dixièmes de millimètres d'épaisseur.

\section{Conclusion}

Bien que plusieurs éléments relatifs aux dernières étapes de la chaîne opératoire de fabrication d'anneaux sur plaquette soient encore incertains, 1'expérimentation nous a permis d'apporter quelques réponses. Nous pouvons noter qu'une cheville centrale en métal offre une meilleure résistance à la pression et que la présence d'une dépouille sur

l'outil permet d'éviter qu'il se coince dans le sillon. La pratique répétée de ce schéma opératoire permettra de dire s'il est valide ou non : il est clair que, la répétition des gestes amenant à une plus grande maîtrise, la réussite en demi-teintes de l'expérimentation a pu tenir de notre inexpérience. Il convient de rappeler que la proposition pré- 
sentée ci-dessus n'est qu'une possibilité de reconstitution de la chaîne opératoire de production d'anneaux sur plaquette et qu'il est probable que d'autres hypothèses puissent faire état de stigmates similaires. D'autres essais sont à prévoir, en incluant les premières étapes de la chaîne opératoire : extraction de la diaphyse puis de la plaque, et mise en forme de celle- $\mathrm{Ci}^{40}$.

\section{Notes}

1. Yehoshua DRAY, "The technology of bone and ivory grafting in Caesarea Maritima, Israel », in Heidi Luik, Alice M. CHoyкe, Colleen E. BAtey et Lembi LõugAs (éd.), From hooves to horns, from mollusc to mammoth: manufacture and use of bone artefacts from Prehistoric Times to the Present : proceedings of the 4th meeting of the ICAZ worked bone research group at Tallinn, 26th-31st of August 2003, Tallinn, université de Tartu (Muinasaja Teadus, 15), 2005, p. 247-252; Mariusz GwiAzda et Bénédicte KHAN, « Luxury craft production on the fringe of the Mediterranean World. Turtle shell objects and production waste from Berenike ", in Steven Sidebotham et Iwona Zych (éd.), Berenike 2012-2013. Report on the excavations in Berenike (Polish Centre of Mediterranean archaeology excavation Series), à paraître; V. J. Hutchinson et D. S. ReEsE, " A worked bone industry at Carthage », in John H. Humphrey (éd.), The circus and a Byzantine cemetery at Carthage, vol. 1, Ann Arbor, University of Michigan Press, 1988, p. 549594; Bénédicte KHAN, "L'artisanat des matières dures animales : nouvelles connaissances à partir des rebuts d'atelier », in Sarah FerJani, Amélie Le Bihan, Marilyne Onfray et Caroline Trémeaud (éd.), Matières premières et gestion des ressources. Actes de la $7^{e}$ Journée doctorale d'archéologie, Paris, 23 mai 2012, Paris, Publications de la Sorbonne (Archeo.Doct., 6), 2014, p. 143-161; François Poplin, "Deux aspects peu connus $\mathrm{du}$ tournage des matières dures d'origine animale dans l'Antiquité ", in Michel Feugère et Jean-Claude Gérold (dir.), Le tournage, des origines à l'an Mil. Actes du colloque international de Niederbronn, 3-6 octobre 2003, Montagnac, Éditions Monique Mergoil (Monographies instrumentum, 27), 2004, p. 127-130; Jacqueline STUDER et Annegret SchneIDER, «Camel use in the Petra
Region, Jordan : 1st Century BC to 4th Century $\mathrm{AD}$ », in Emmanuelle Vila, Lionel Gourichon, Alice M. Сноуке et Hijlke Buitenhuis (éd.), Archaeozoology of the Near East VIII : Actes des huitièmes rencontres internationales d'archéozoologie de l'Asie du Sud-Ouest et des régions adjacentes, t. II, Lyon, Maison de l'Orient et de la Méditerranée (Travaux de la Maison de l'Orient et de la Méditerranée, 49), 2008, p. 581-596.

2. J. Studer et A. Schneider, " Camel use in the Petra Region... », op. cit., p. 590.

3. Cette hypothèse s'inspire des bourses médiévales à deux poches que l'on mettait à la ceinture (C. Picod, communication personnelle).

4. C. Bouchaud, communication personnelle. Des exemples de quenouilles d'époque romaine en verre à deux anneaux dont l'un, mobile, montait et descendait le long de la tige, appuient cette hypothèse (C. Picod, communication personnelle) ; [http://artefacts.mom.fr/fr/libraries/ tcpdf/examples/essai6.php?id=QNL-4004)]. Mais lorsque des quenouilles à anneaux en os ont été mises au jour, aucun anneau mobile n'y était, à notre connaissance, lié. D'autres hypothèses sont proposées par E. Ayalon : parties d'objets composites, élément de placage, etc., Etan Aralon, The assemblage of bone and ivory artefacts from Caesarea Maritima, Israel 1st-13th Centuries CE, Oxford, Archeopress (British archaeological reports, International Series, 1457), 2005.

5. F. Poplin, « Deux aspects peu connus du tournage des matières dures d'origine animale dans l'Antiquité ", op. cit.; Y. DrAY, "The technology of bone and ivory grafting in Caesarea Maritima, Israel ", op. cit.; J. Studer et A. SChNeIDER, "Camel use in the Petra Region, Jordan... ", op. cit., p. 590, Fig. 9.

6. B. KHAN, « L'artisanat des matières dures animals... », op. cit., p. 156-157.

7. Les sources antiques sont relativement silencieuses sur le travail des matières dures animales. Quelques informations sont données sur des points précis quant à l'ivoire, l'écaille de tortue ou la corne (Pline, Histoire naturelle, XVI.84.3, entre autres), mais pas sur le travail de l'os.

8. Aline Averboun, Technologie de la matière osseuse travaillée et implications palethnologiques. L'exemple des chaînes d'exploitation du bois de cervidé chez les Magdaléniens des Pyrénées, thèse inédite, université de Paris 1 Panthéon-Sorbonne, 2000; Noëlle Provenzano, Les industries en os et en bois de cervidés des terramares émiliennes, thèse inédite, université de Provence-Centre d'Aix, 2001.

9. John William Humphrey, John Peter Oleson et Andrew Neil SHERwood, Greek and roman 
technology : a sourcebook, Londres et New York, Routledge, 1998, p. 337.

10.Ibid., p. 337 et 343 ; E. Ayalon, The assemblage of bone and ivory artefacts from Caesarea Maritima..., op. cit., p. 132.

11. Aline Averboun et Noëlle Provenzano, «Propositions pour une terminologie du travail préhistorique des matières osseuses : 1- Les techniques ", Préhistoire anthropologie méditerranéennes, $\mathrm{n}^{\circ}$ 7-8, 1998-1999, p. 5-25.

12. Ainsi, le raclage n'est pas forcément effectué à l'aide d'un racloir, mais peut être fait avec tout " outil dont la partie active possède une arête [...] aigüe » (Ibid., p. 14) : herminette, plane, ciseau, etc.

13. H. Monchot, communication personnelle.

14. M. Gwiazda et B. KHAN, "Luxury craft production on the fringe of the Mediterranean World... ", op. cit.

15. Des rejets en corne de bovidé ont été retrouvés à Béréniké dans les mêmes dépôts secondaires que les éléments en écaille de tortue et portant des stigmates similaires. Une étude est en cours sur ces éléments.

16. Même si des outils ont été retrouvés en contexte artisanal, il n'est pas certain qu'ils aient été utilisés uniquement pour le travail des matières dures animales. Ainsi, bien qu'une scie ait été retrouvée à Maastricht avec des éléments de bois de cervidés travaillés, les auteurs n'excluent pas le fait qu'elle a pu être liée à d'autres activités artisanales : Wim DiJKMAn et Anton ERvYNCK, Antler, bone, horn, ivory and teeth : the use of animal skeletal materials in Roman and Early Medieval Maastricht, Maastricht, Department of urban development and ground maintenance (Archaelogica Mosana, 1), 1998, p. 17.

17. Richard David BARNETT, Ancient ivories in the Middle East, Jérusalem, Hebrew University, Institute of archaeology (Qedem, 14), 1982, p. 70; E. Ayalon, The assemblage of bone and ivory artefacts from Caesarea Maritima..., op. cit., p. 131.

18. Paula WAPNISH, "Bone, ivory and shell", in Eric M. Meyers (éd.), The Oxford Encyclopedia of archaeology of the Near East, vol. 1, Abba-Chue, New York et Oxford, Oxford University Press, 1997, p. 335-350.

19. C'est d'ailleurs le cas à Pompéi, Nicolas MonteIx, «De "l'artisanat" aux métiers. Quelques réflexions sur les savoir-faire du monde romain à partir de l'exemple pompéien ", in Nicolas Monteix et Nicolas Tran (dir.), Les savoirs professionnels des gens de métier. Études sur le monde du travail dans les sociétés urbaines de l'empire romain, Naples, Centre Jean Bérard (Collection du Centre Jean Bérard, 37; Archéologie de l'artisanat antique, 5), 2011, p. 7-26.
20. J. W. Humphrey et al., Greek and roman technology : a sourcebook, op. cit., p. 343-344; Roger Bradley ULRICH, Roman woodworking, New Haven et Londres, Yale University Press, 2007.

21. Pline, Histoire naturelle, VII.57.7; Anthologie grecque, traduite sur le texte publié d'après le manuscrit palatin par Fr. Jacobs, avec des notices sur les poètes de l'Anthologie. Tome premier, Paris, Hachette, 1863 , p. $85,100-101, \mathrm{n}^{\circ} 103,204-205$.

22. Jean-Claude GÉrold, "Réalisation d'un trépan à archet ", Bulletin Instrumentum, $\mathrm{n}^{\circ} 12$, 2000 , p. $27-28$

23. Jean-Claude BÉAL, « Tabletterie et tabletiers d'os en Gaule romaine ", in Aurochs, le retour : aurochs, vaches et autres bovins de la préhistoire à nos jours, Lons-le-Saunier, Centre jurassien du patrimoine, 1994, p. 121-130.

24. Pline, Histoire naturelle, VII.57.7.

25. Alexandros Emm. Kontōleon, " Epigraphika ", Mitteilungen des Deutschen Archäologischen Instituts, Athenische Abteilung, $\mathrm{n}^{\circ} 15,1890$, p. 330337. Pour d'autres exemples de tours, voir Pierre Mille, «Inventaire des différents types de tours utilisés en Europe occidentale, des origines à l'époque médiévale, d'après la documentation textuelle, archéologique et iconographique ", in M. Feugère et J.-C. Gérold (dir.), Le tournage, des origines à l'an Mil..., op. cit., p. 17-26.

26. L'objet en question a été inventorié comme trépan. Renseignements : Conservation départementale d'archéologie de la Moselle/Parc archéologique européen de Bliesbruck-Reinheim.

27. Ernst KüNZL, Medizinische Instrumente aus Sepulkralfunden der römischen Kaiserzeit, Cologne, Rheinland-Verl (Kunst und Altertum am Rhein, 115), 1983, p. 80 et 84, Fig. 58-59.

28 . Une proposition de reconstitution du fonctionnement de cet objet, fondée sur la découverte de Bingen où le trépan présente une pointe en son centre (E. Künzl, op. cit., Fig. 58), démontre la nécessité d'une perforation centrale conique non traversante qui ne serait utilisée que pour centrer l'amorce du sciage circulaire (C. Picod, communication personnelle). Il n'y aurait pas besoin d'un élément de calage central comme dans le matériel du Qasr al-Bint et de Béréniké.

29. Des anneaux de diamètres plus réduits, datant d'entre le Hallstatt final et la Tène finale, ont été travaillés à l'aide d'un tel outil tripartite à Aulnat : Delphine MinNI, « Le tournage du bois, des lignites, de l'ambre et des matières dures d'origine animale à l'Âge du Fer ", dans M. FeugÈre et J.-C. Gérold (dir.), Le tournage, des origines à l'an Mil..., op. cit., p. 113-126.

30. L'idée d'une pointe pour travailler la matière n'est pas viable : elle s'émousse vite et se coince dans le sillon. 
31. Voir, par exemple, Description de l'Égypte ou recueil des observations et des recherches qui ont été faites en Égypte pendant l'expédition de l'armée française, seconde édition, État Moderne, tome deuxième, Paris, C. L. F. Panckoucke, 1823, Pl. XV, Fig. 4; André Leroi-Gourhan, L'homme et la matière, Paris, Albin Michel, 1943 (rééd. 1971), p. 181, Fig. 320.

32. Christophe PICOD, « À propos du tournage à l'époque romaine : un tour et un médaillon en bois de cerf », Bulletin Instrumentum, n ${ }^{\circ} 17,2003$, p. 24-25.

33. A. Averbouh et N. Provenzano, «Propositions pour une terminologie du travail préhistorique des matières osseuses : 1- Les techniques », op. cit., p. 15.

34. Barbara Armbruster, « Le tournage dans l'orfèvrerie de l'Âge et du premier âge du Fer en Europe Atlantique ", in M. Feugère et J.-C. GÉROld (dir.), Le tournage, des origines à l'an Mil..., op. cit., p. 53-70.

35. Ibid., p. 55.

36. Il est évident que ces méthodes de dégraissage, très modernes, ont pu avoir un impact sur la manière dont la matière allait répondre par la suite, lors de l'expérimentation. Il était cependant extrêmement compliqué à ce moment-là de mener une expérimentation de l'acquisition de la matière première à l'obtention de l'anneau. Une telle expérimentation est néanmoins prévue.

37. Une description détaillée du tour est donnée dans Christophe PICOD, " Tournage expérimental à l'archet de jetons en os ", Bulletin Instrumentum, $\mathrm{n}^{\mathrm{o}} 12,2000$, p. 25-26. Il est probable que le tour reconstitué ne soit pas exactement comme ceux de l'époque romaine. De plus, l'objet a été travaillé en position debout, alors que les exemples ethnologiques montrent des artisans assis devant le tour à archet (Description de l'Égypte..., op. cit. ; A. LEROIGourhan, L'homme et la matière, op. cit.). La position assise est également récurrente dans les représentations médiévales (voir le patenôtrier du Mendelsches Brüderbuch, ou le fabriquant de pièces de jeux d'échec dans le Livre des Jeux d'Alphonse X le Sage)

38. Ces pointes métalliques ont été utilisées lors du tournage expérimental de couronnes en bois de cerf : C. PICOD, « À propos du tournage à l'époque romaine », op. cit., p. 25. Il est possible que ces pointes ne soient pas nécessaires pour la production d'anneaux; l'utilisation d'une colle (C. PICOD, « Tournage expérimental à l'archet de jeton en os », op. cit., p. 25-26) en plus de la cheville, voire la cheville seule, peut avoir suffi au maintien et à la stabilité de la plaquette sur le mandrin. Cela reste à démontrer.

39. Edgard O. EspinozA, Barry W. BAKER et Craig A. BERRY, "The analysis of sea turtle and bovid keratin artefacts using drift spectroscopy and discriminant analysis ", Archaeometry, n 49-4, 2007, p. 685-698.

40. M- Barbier avance une hypothèse de travail pour ces étapes, Marc BARBIER, L'artisanat de l'os à l'époque gallo-romaine. De l'ostéologie à l'archéologie expérimentale, Oxford, Archaeopress (Archaeopress Roman archaeology, 16), 2016, p. 19-20. Néanmoins, certaines propositions ne rejoignant pas les stigmates relevés sur le matériel archéologique étudié, il s'avère pertinent de reprendre les premières phases de la chaîne opératoire de production de plaquettes. 